\title{
SIRT3 is attenuated in systemic sclerosis skin and lungs, and its pharmacologic activation mitigates organ fibrosis
}

\author{
Kaname Akamata ${ }^{1, *}$, Jun Wei ${ }^{1, *}$, Mitra Bhattacharyya ${ }^{1}$, Paul Cheresh $^{2}$, Michael Y. \\ Bonner $^{3}$, Jack L. Arbiser ${ }^{3,4}$, Kirtee Raparia5, Mahesh P. Gupta6, David W. Kamp ${ }^{2,7}$ \\ and John Varga ${ }^{1}$ \\ ${ }^{1}$ Division of Rheumatology, Feinberg School of Medicine, Northwestern University, Chicago, IL, USA \\ 2 Division of Pulmonary \& Critical Care Medicine, Feinberg School of Medicine, Northwestern University, Chicago, IL, USA \\ ${ }^{3}$ Department of Dermatology, Emory University School of Medicine, Atlanta, GA, USA \\ ${ }^{4}$ Atlanta Veterans Administration Medical Center and Winship Cancer, Atlanta, GA, USA \\ ${ }^{5}$ Department of Pathology, Northwestern University, Chicago, IL, USA \\ ${ }^{6}$ Department of Surgery, University of Chicago, Chicago, IL, USA \\ 7 Jesse Brown VA Medical Center, Chicago, IL, USA \\ * These authors have contributed equally to this work \\ Correspondence to: John Varga, email: j-varga@northwestern.edu
}

Keywords: fibrosis, SIRT3, TGF- $\beta$, myofibroblast, ROS, Pathology Section

Received: July 10,2016 Accepted: September 29, $2016 \quad$ Published: October 06, 2016

\section{ABSTRACT}

Constitutive fibroblast activation is responsible for organ fibrosis in fibrotic disorders including systemic sclerosis (SSc), but the underlying mechanisms are not fully understood, and effective therapies are lacking. We investigated the expression of the mitochondrial deacetylase sirtuin 3 (SIRT3) and its modulation by hexafluoro, a novel fluorinated synthetic honokiol analogue, in the context of fibrosis. We find that augmenting cellular SIRT3 by forced expression in normal lung and skin fibroblasts, or by hexafluoro treatment, blocked intracellular TGF-ß signaling and fibrotic responses, and mitigated the activated phenotype of SSc fibroblasts. Moreover, hexafluoro attenuated mitochondrial and cytosolic reactive oxygen species (ROS) accumulation in TGF- $\beta$-treated fibroblasts. Remarkably, we found that the expression of SIRT3 was significantly reduced in SSc skin biopsies and explanted fibroblasts, and was suppressed by TGF- $\beta$ treatment in normal fibroblasts. Moreover, tissue levels of acetylated MnSOD, a sensitive marker of reduced SIRT3 activity, were dramatically enhanced in lesional skin and lung biopsies from SSc patients. Mice treated with hexafluoro showed substantial attenuation of bleomycin-induced fibrosis in the lung and skin. Our findings reveal a cell-autonomous function for SIRT3 in modulating fibrotic responses, and demonstrate the ability of a novel pharmacological SIRT3 agonist to attenuate fibrosis in vitro and in vivo. In light of the impaired expression and activity of SIRT3 associated with organ fibrosis in SSc, pharmacological approaches for augmenting SIRT3 might have therapeutic potential.

\section{INTRODUCTION}

Fibrosis, defined by the excessive accumulation of the extracellular matrix (ECM) in inflamed tissue, leads to permanent scarring and organ malfunction [1]. Systemic sclerosis (SSc) is characterized by widespread fibrosis, accompanied by autoimmunity and vasculopathy
[2]. Greater than $50 \%$ of patients with $\mathrm{SSc}$ die, or develop organ failure, within ten years, with mortality due in large part to fibrosis in the lungs [3]. Fibrosis is caused by activation of myofibroblasts triggered and then perpetuated by transforming growth factor- $\beta$ (TGF- $\beta$ ) and related growth factors, cytokines, peptides, hypoxia and stiffening of the matrix [4]. Transforming growth factor- $\beta$ 
also stimulates production of reactive oxygen species (ROS) from mitochondrial and cytosolic enzymes, which leads to cellular oxidative stress and directly contributes to myofibroblast differentiation and fibrosis progression $[5,6]$.

Sirtuins (SIRTs), the mammalian orthologs of yeast silent mating-type information regulator 2 (Sir2), are NAD-dependent class III deacetylases with pleiotropic effects on metabolism, cell survival, cancer, processes of aging, and calorie restriction-mediated longevity in organisms ranging from yeast to human [79]. The most extensively studied member of the family, SIRT1, is principally nuclear, while SIRT3 is located within mitochondria and implicated in mitochondrial homeostasis [10, 11]. Fibroblasts lacking SIRT3 exhibit aberrant glucose metabolism and generate increased levels of ROS when exposed to oxidative stress, genotoxic stress, and ionizing radiation [12]. Using genome-wide transcriptome profiling, we previously showed that treatment of explanted healthy and SSc fibroblasts with TGF- $ß$ resulted in down-regulation of SIRT3 expression [13]. Of interest, a recent study showed that pharmacologic activation or forced expression of SIRT3 attenuated alpha-smooth muscle actin ( $\alpha$-SMA) and collagen gene expressions in a mouse model of cardiac hypertrophy [14].

Mitochondria are a significant source of ROS such as the superoxide radical $\left(\mathrm{O}_{2}^{-}\right)$that can react with DNA and other biomolecules to induce mutagenesis, senescence, and ultimately cell death [15]. The primary mitochondrial mechanism for maintaining physiological $\mathrm{O}_{2}^{-}$involves manganese superoxide dismutase (MnSOD) [16]. Acetylation of mitochondrial MnSOD catalyzed by SIRT3 plays a pivotal role in modulating its enzymatic activity, and down-regulation of SIRT3 is associated with enhanced levels of acetylated MnSOD in tissues [17-19].

Honokiol (HNK) [2-(4-hydroxy-3-prop-2-enylphenyl)-4-prop-2-enyl-phenol], a small molecular weight biphenolic compound derived from the magnolia tree bark, has anti-thrombotic, anti-inflammatory, anti-tumor, cytoprotective and anti-oxidative activities, suggesting a potential therapeutic utility in a variety of chronic conditions [20-23]. A recent study showed that HNK induced SIRT3 in cardiomyocytes in vitro and in vivo [14]. In an effort to generate more lipophilic HNK derivatives with enhanced drug-like properties, hexafluoro (bistrifluoromethyl-bis-(4-hydroxy-3-allylphenyl) methane) was synthesized and shown to have activity against vemurafenib-resistant melanoma cells in vivo [24].

Little is currently known regarding the expression and function of SIRT3 in fibroblasts, or their potential role in fibrosis in SSc. Moreover, the modulation of SIRT3 by HNK derivatives in this context is not understood. Our present results show impaired expression and activity of SIRT3 in skin and lung biopsies from patients with SSc. In normal fibroblasts, TGF- $\beta$ treatment caused downregulation of cellular SIRT3, while hexafluoro rescued
SIRT3 expression in these cells. Moreover, hexafluoro abrogated TGF- $\beta$-induced stimulation of fibrotic gene expression, myofibroblast differentiation, and cellular ROS production in normal fibroblasts. In vivo, hexafluoro mitigated bleomycin-induced lung and skin fibrosis in the mouse. These results implicate aberrant SIRT3 function in the pathogenesis of SSc, and suggest that pharmacological enhancement of SIRT3 may represent a promising novel therapeutic approach.

\section{RESULTS}

\section{SIRT3 negatively regulates fibrotic responses}

Excessive mitochondrial ROS production is implicated in the pathogenesis of fibrosis in multiple organs $[25,26]$. As a mitochondrial deacetylase, SIRT3 has a vital function in maintaining mitochondrial integrity and modulating oxidative stress [27]. To examine the potential role of SIRT3 in controlling fibrotic responses, we used gain-of-function and loss-of-function experiments. Ectopic expression of SIRT3 in normal lung fibroblasts was sufficient to suppress TGF- $\beta$-induced stimulation of collagen synthesis (Figure 1A and 1B). It was of interest to delineate the intracellular pathways targeted by SIRT3 in these cells, focusing on canonical Smad signaling, the principal mechanism for fibrotic signaling [4]. Transient transfection assays demonstrated that TGF- $\beta$ stimulation of a Smad-responsive [SBE] $]_{4}$-luc reporter in fibroblasts was significantly reduced by SIRT3 overexpression (Figure 1C). Conversely, even partial down-regulation of cellular SIRT3 by RNAi in normal lung fibroblasts resulted in spontaneous elevation of multiple fibrotic genes (Figure 1D). Comparable results were seen using skin fibroblasts from healthy adult donors (Suppl. Fig 1). Collectively, these results indicate that in fibroblasts SIRT3 serves as a cell-autonomous negative regulator to constrain fibrotic gene expression.

\section{Hexafluoro enhances SIRT3 expression}

In light of SIRT3's anti-fibrotic effects, we speculated that it may have a physiologic regulatory role in maintaining fibroblast homeostasis, and that failure of this regulatory function could contribute to pathologic fibrosis. To begin to explore this concept, we used a novel pharmacological analogue of honokiol, a naturally-occurring biphenolic compound derived from the magnolia tree bark that was recently shown to be a SIRT3 activator [24]. The novel honokiol derivative hexafluoro (Figure 2A, upper panel) was synthesized by adding bis-trifluoro methyl radicals to honokiol [24] with better lipophilic characteristics. Incubation of confluent fibroblasts with hexafluoro resulted in time- and dose- 
A

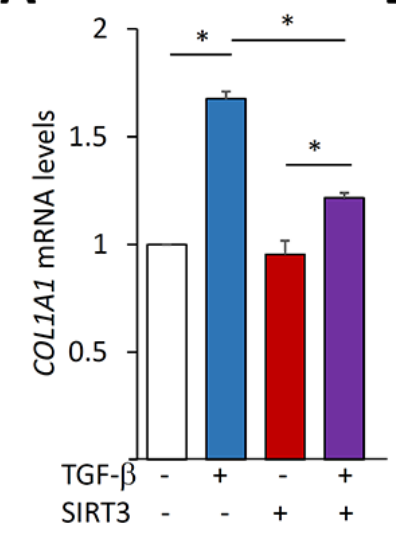

B

D

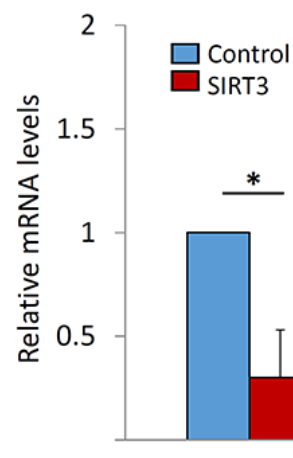

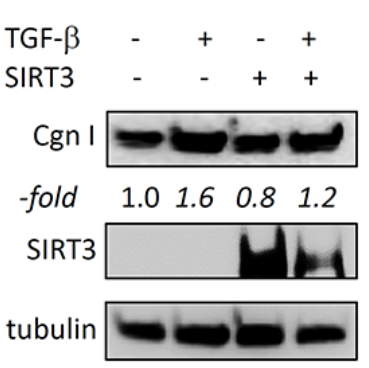

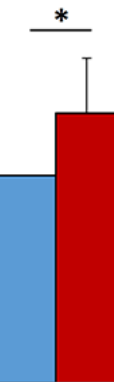

COL1A1
C

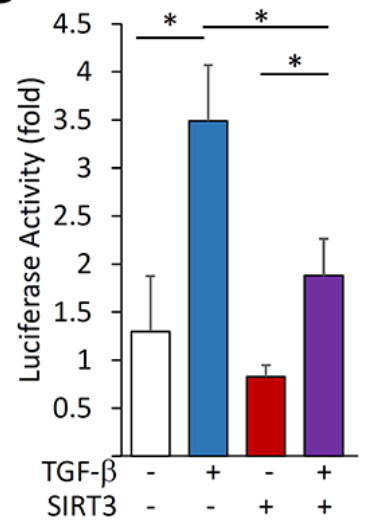

SIRT3

Figure 1: SIRT3 negatively regulates fibrotic responses. A.-C. Normal adult lung fibroblasts transfected with SIRT3 or empty vector were incubated with TGF- $\beta 2(10 \mathrm{ng} / \mathrm{ml})$ for $24 \mathrm{~h}$. A. Results of real-time qPCR normalized with GAPDH are means \pm SD of triplicate determinations from an experiment representative of three. ${ }^{*} p<0.05$. B. Whole cell lysates were examined by Western analysis. Representative blots from an experiment representative of three; -fold change in band intensities normalized with tubulin shown below. Cgn I, Type I collagen. C. Fibroblasts were co-transfected [SBE] -luc along with SIRT3 or empty vector. Whole cell lysates were analyzed for their luciferase activities. Results are the means $\pm \mathrm{SD}$ of triplicate experiments. ${ }^{*} p<0.05$. D. Fibroblasts were transiently transfected with SIRT3 siRNA or scrambled (control) siRNA and incubated for $48 \mathrm{~h}$. Results of qRT-PCR normalized with GAPDH are means \pm SD of triplicate determinations from an experiment representative of three. ${ }^{*} p<0.05$.

A
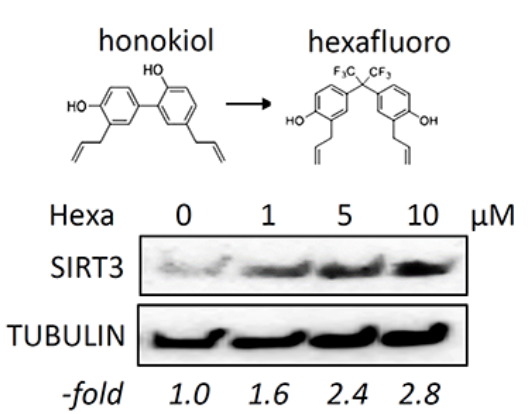

B

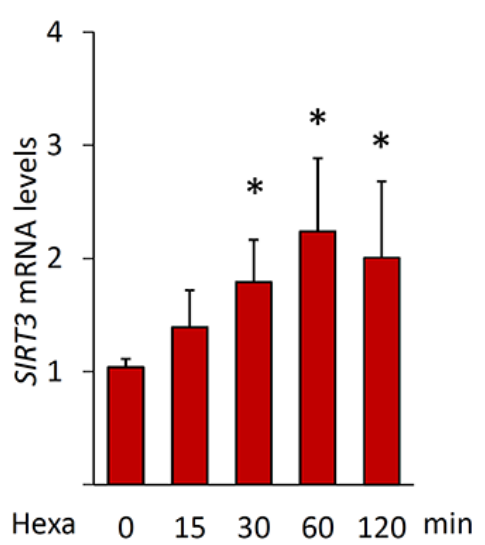

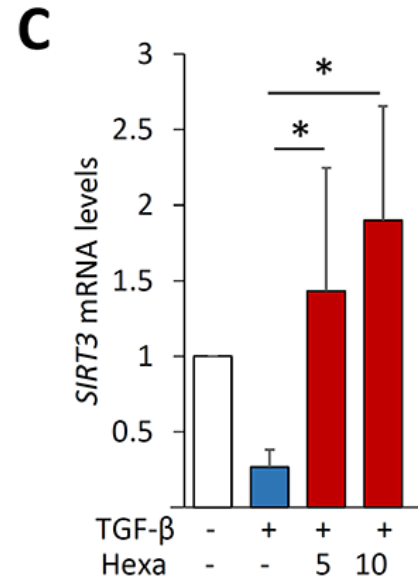

Figure 2: Hexafluoro stimulates SIRT3 expression. Lung fibroblasts were incubated with hexafluoro (10 $\mu \mathrm{M}$ or indicated concentrations) for $24 \mathrm{~h}$ or indicated periods, in the absence or presence of TGF- $\beta 2$ ( $10 \mathrm{ng} / \mathrm{ml}$ and indicated times). A. Whole cell lysates were examined by Western analysis. Representative blots. - fold change in band intensities normalized with tubulin shown below. B., C. Real-time qPCR results normalized with $G A P D H$ are means \pm SD of triplicate determinations from an experiment representative of three. $*, p<0.05$. 
dependent augmentation of SIRT3, while no increase in SIRT1 was seen (Figure 2B and data not shown). Trypan blue dye exclusion and LDH assays showed that cell viability was unaffected by hexafluor in concentrations up to $10 \mu \mathrm{M}$ of hexafluoro (data are not shown). Treatment of human lung fibroblasts with TGF- $\beta$ caused a significant decrease in SIRT3 mRNA and protein levels (Figure 2C). Skin fibroblasts showed a comparable downregulation of SIRT3 (Suppl. Figs. 2A and B, and data not shown). Remarkably, pre-incubation of the cultures with hexafluoro for $30 \mathrm{~min}$ rescued SIRT3 mRNA expression even in the presence of TGF- $\beta$ (Figure $2 \mathrm{C}$ ).

\section{Hexafluoro blocks TGF- $\beta$-induced fibrotic responses.}

In order to further explore the modulation of fibroblast responses, confluent lung fibroblasts were pre- incubated with hexafluoro $(10 \mu \mathrm{M})$ for $30 \mathrm{~min}$ prior to TGF- $\beta$ (10 ng/ml), and harvested following a further 24 $\mathrm{h}$ incubation. Treatment with TGF- $\beta$ induced a significant increase in the expression of fibrotic gene such as COL1A1, COL1A2, $\alpha$ SMA and fibronectin EDA $\left(\mathrm{FN}^{\mathrm{EDA}}\right)$, and in myofibroblast differentiation, as expected, while hexafluoro pretreatment dose-dependently suppressed these fibrotic responses accompanied by increase in levels of SIRT3 (Figure 3A-3C). Significantly, hexafluoro exerted comparable anti-fibrotic effects in skin fibroblasts (Suppl. Figs 2C, D). Moreover, abrogation of TGF- $\beta$ induced fibrotic responses by hexafluoro was seen even when hexafluoro added after TGF- $\beta$ (Suppl. Fig 2E). To investigate the effects of hexafluoro on tissue remodeling, in vitro wound healing and collagen lattice gel contraction assays were performed [28]. Incubation with hexafluoro significantly attenuated TGF- $\beta$-induced stimulation of both fibroblast contractility (Figure 4A) and migration (Figure 4B). Collectively, these results indicate that

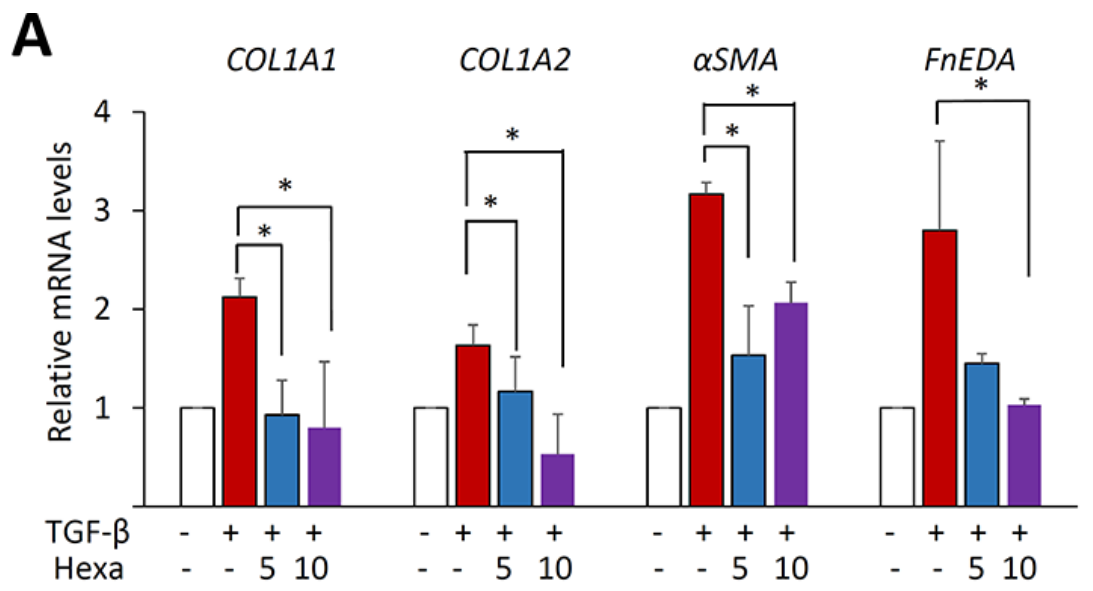

B
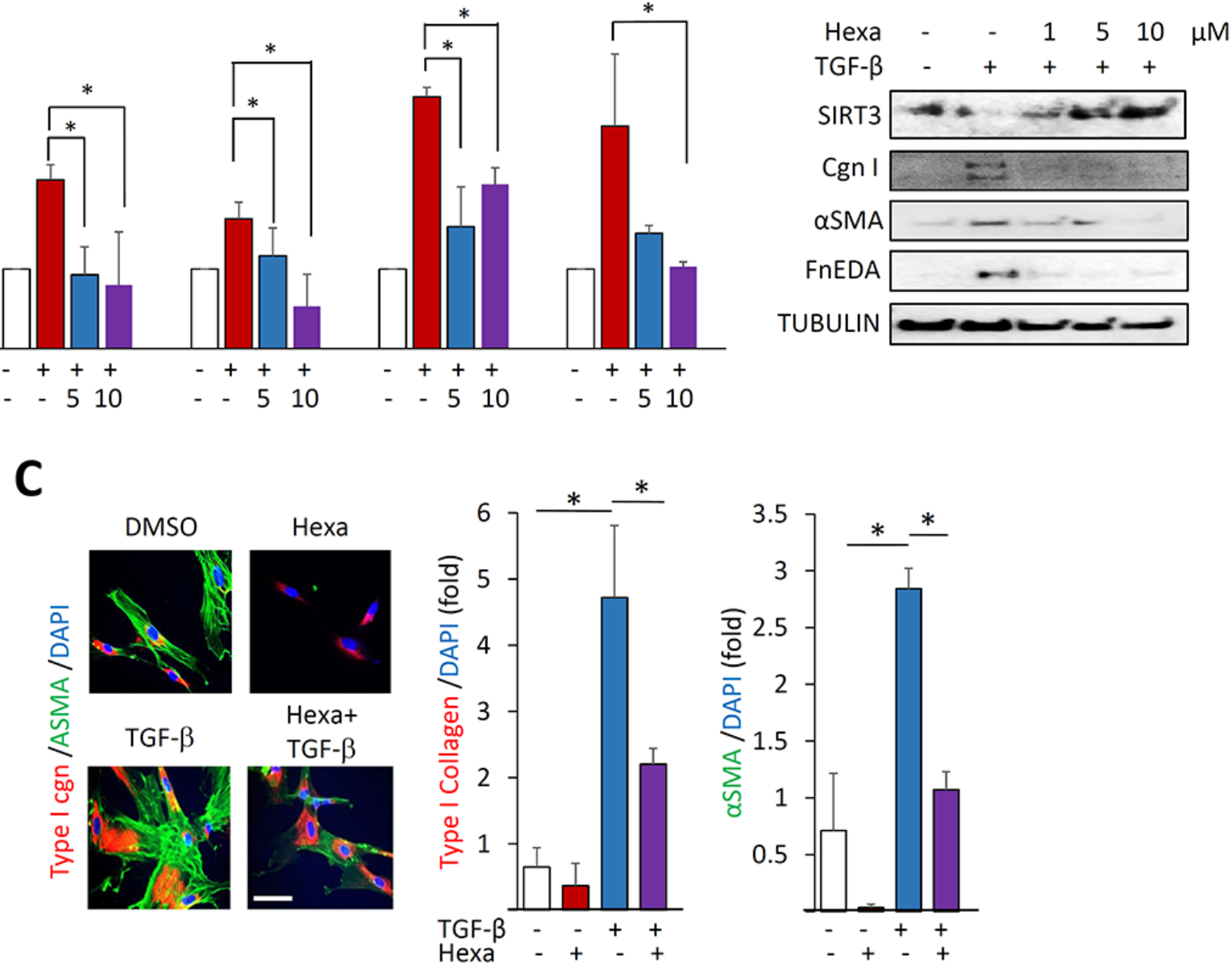

Figure 3: Hexafluoro attenuates TGF- $\beta$-induced fibrotic gene expression. Lung fibroblasts were preincubated with hexafluoro $(10 \mu \mathrm{M})$ for $30 \mathrm{~min}$, followed by TGF- $\beta 2(10 \mathrm{ng} / \mathrm{ml})$ for $24 \mathrm{~h}$. A. Relative mRNA levels were examined by real-time qPCR. Results normalized with $G A P D H$ are means \pm SD of triplicate determinations from a representative experiment of three. *, $p<0.05$. B. Whole cell lysates were examined by Western analysis. Representative images. Cgn I, Type I collagen. C. Left, Confocal immunofluorescence using antibodies to $\alpha$ SMA (green) and Type I collagen (red). Bar $=10 \mu \mathrm{m}$. Right, Quantification of immunofluorescence intensity. Results are the means $\pm \mathrm{SD}$ of triplicate experiments. 
hexafluoro potently inhibits the induction of fibrotic gene expression, myofibroblast differentiation, contraction and migration in normal fibroblasts.

\section{Hexafluoro mitigates ROS enhanced production} and changes in mitochondrial membrane potential

Myofibroblast differentiation in response to TGF- $\beta$ is associated with, and indeed dependent on, cytosolic as well as mitochondrial ROS production and changes in mitochondrial membrane potential $[29,30]$. We therefore examined the effects of hexafluoro on alterations in mitochondrial function in the context of TGF- $\beta$ induced myofibroblast differentiation. As expected, TGF- $\beta$ treatment resulted in enhanced mitochondrial ROS production, which was markedly attenuated when fibroblasts were pre-treated with hexafluoro (Figure 5A). Enhanced cytosolic accumulation of ROS was similarly attenuated (Figure 5B). Next, fibroblasts were loaded with TMRM, a mitochondrial membrane potential-sensitive

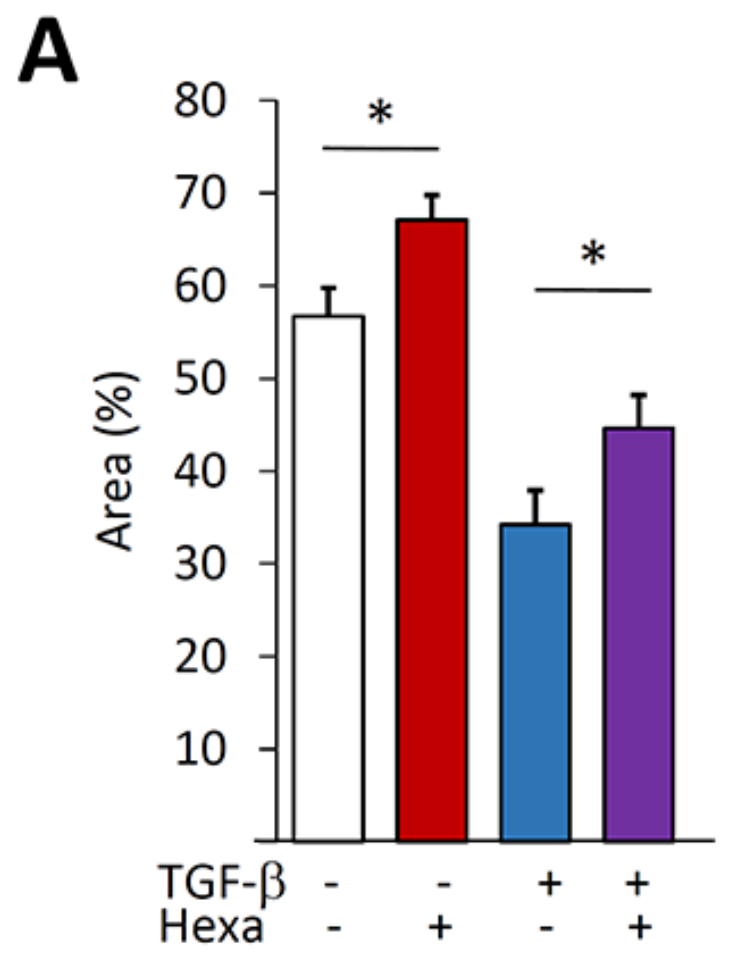

B
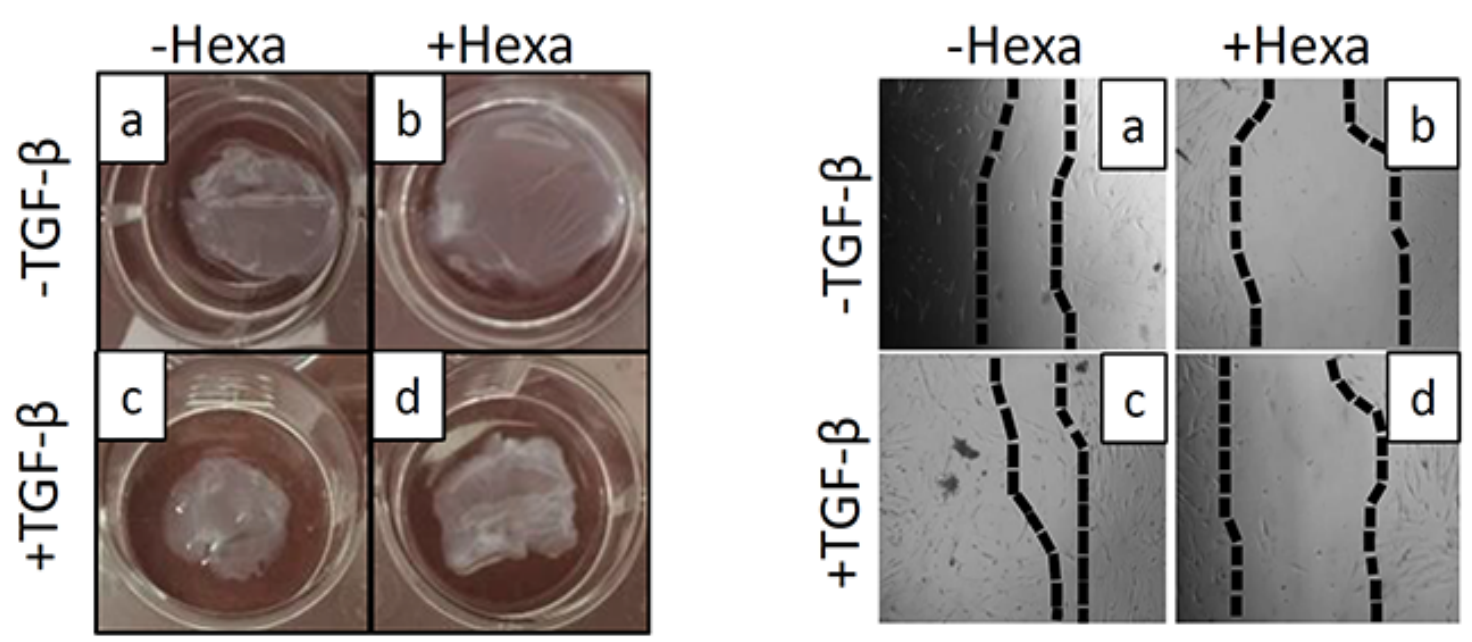

Figure 4: Hexafluoro attenuates TGF- $\beta$-induced cell contraction and migration. Lung fibroblasts were pretreated with $10 \mu \mathrm{M}$ hexafluoro for $30 \mathrm{~min}$, followed by TGF- $\beta 2(10 \mathrm{ng} / \mathrm{ml})$ for further 72 (A) and $48 \mathrm{~h}$ (B). A. Gel contraction assays $(n=3)$. Representative images. Results are means \pm SD of triplicate experiments. ${ }^{*} p<0.05$. B. Wound healing assays $(n=3)$. Representative images. Results are means \pm SD of triplicate experiments. ${ }^{*} p<0.05$. 
fluorescent dye. While treatment with TGF- $\beta$ was associated with the appearance of perinuclear red punctae that are indicative of active polarized mitochondria, the uptake of TMRM was substantially attenuated in the presence of hexafluoro (Figure 5C, 5D). Taken together, these results indicate that in TGF- $\beta$-treated normal fibroblasts hexafluoro exerts mitochondrial-protective effects.

\section{Hexafluoro blocks activation of Smad2/3 and Stat3.}

To investigate the mechanism underlying the anti-fibrotic effects of hexafluoro, we focused on the canonical Smad signal transduction pathway fundamental for fibrotic responses [31]. We found that Smad2 phosphorylation and nuclear translocation induced by TGF- $\beta$ in normal fibroblasts were markedly attenuated by hexafluoro (Figure 6A). Moreover, the magnitude of Smad-dependent transcriptional responses was reduced in a dose-dependent manner (Figure 6B). Hexafluoro also attenuated TGF- $\beta$-induced phosphorylation of STAT3, a key mediator of fibrotic IL-6 signaling [32] and a potential effector in pathological fibrosis (Figure 6C). To examine the cell-autonomous role of SIRT3 as a negative regulator mediating the anti-fibrotic effects of hexafluoro, we examined fibrotic responses in fibroblasts lacking endogenous SIRT3. The results indicated that both basal and TGF- $\beta$-stimulated levels of $\alpha$ SMA were elevated in SIRT3-- MEFs. Moreover, these cells were partially resistant to the anti-fibrotic effects of hexafluoro, indicating that these inhibitory effects were at least in part SIRT3-dependent (Figure 6D).

\section{Hexafluoro ameliorates experimentally-induced organ fibrosis in mouse}

In light of the robust, and at least partially SIRT3dependent, anti-fibrotic effects of hexafluoro in fibroblasts, it was of interest to assess the impact of hexafluoro
A
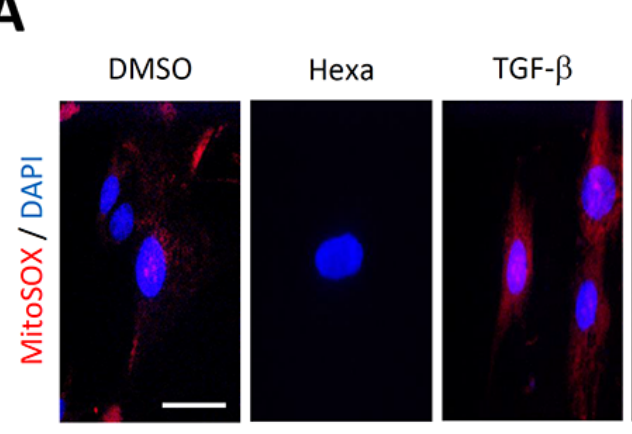

C

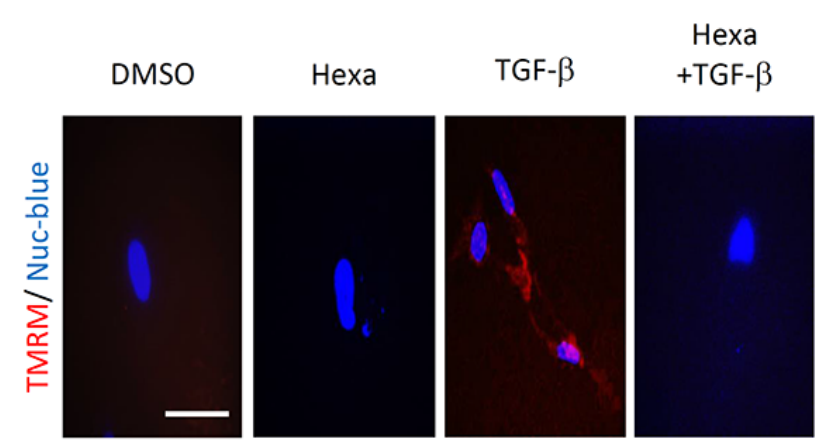

B

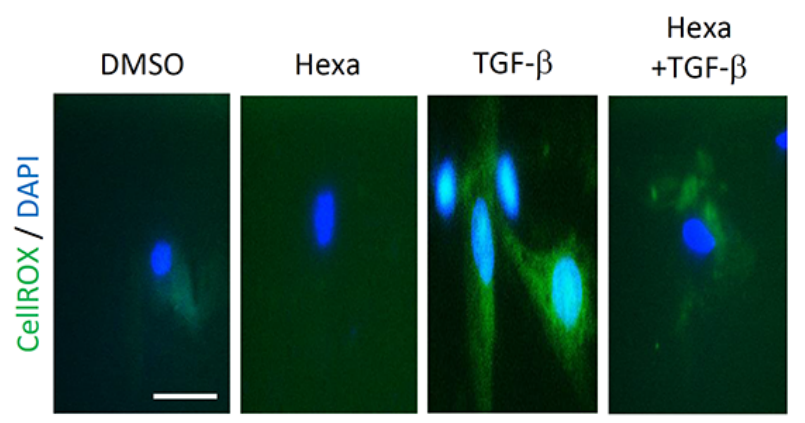

D

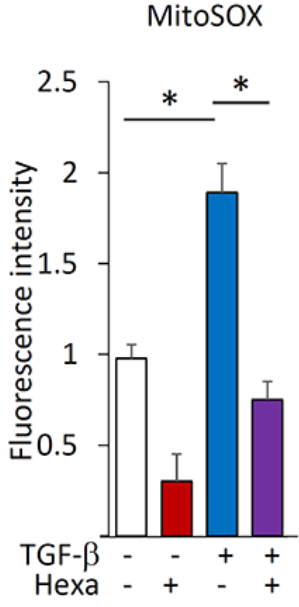

CellROX

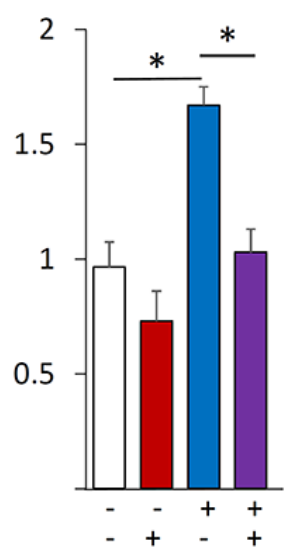

TMRM

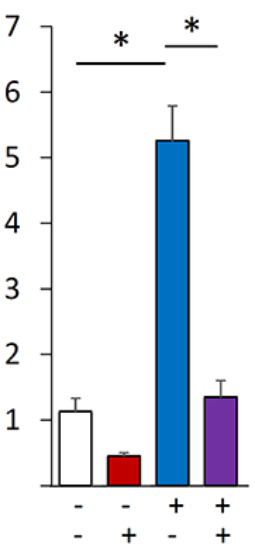

Figure 5: Hexafluoro abrogates TGF- $\beta$-induced ROS production and mitochondrial membrane potential. Lung fibroblasts were preincubated with $10 \mu \mathrm{M}$ hexafluoro for $30 \mathrm{~min}$, followed by TGF- $\beta 2(10 \mathrm{ng} / \mathrm{ml})$ for $24 \mathrm{~h}$. A., B. Mitochondrial ROS (red) determined using MitoSOX Red Mitochondrial Superoxide indicator, and cytosolic ROS (green) determined using CellROX Green. Cells were counterstained with DAPI (blue). Bar $=10 \mu \mathrm{m}$. C. Cultures stained with Nuc-blue (blue) and TMRM fluorescent dyes (red). Bar $=$ $10 \mu \mathrm{m}$. D. Quantification of MitoSOX, CellROX and TMRM fluorescence. Bars represent means \pm SD of triplicate determinations. ${ }^{*} p<$ 0.05 . 
treatment in a bleomycin-induced model of multiple-organ fibrosis [33]. Female 8-10 week-old C57BL/6J mice were randomized to treatment with vehicle, s.c. bleomycin, or a combination of s.c. bleomycin plus i.p. hexafluoro (70 $\mathrm{mg} / \mathrm{kg} /$ day). In these experiments, hexafluoro for up to 28 days was well tolerated, and treatment was not associated with significant weight loss, behavioral changes or other signs of toxicity. Bleomycin given s.c. induced prominent changes in the lungs (Figure 7A). Pulmonary fibrotic pathology was substantially attenuated in mice treated with hexafluoro (Figure 7A, right panel). Bleomycintreated mice also developed skin fibrosis and increased collagen accumulation that were significantly ameliorated by hexafluoro (Figure 7B, 7C). Together, these results showed that hexafluoro has potent protective effects on both lung and skin fibrosis in a bleomycin-induced mouse model.

\section{SIRT3 expression and function are decreased in $\mathrm{SSc}$ and in fibrotic tissues in the mouse}

The potent in vitro and in vivo anti-fibrotic effects of hexafluoro led us to speculate that its target, SIRT3, itself might play a regulatory role in pathological fibrosis in SSc, and its dysfunction might contribute to disease
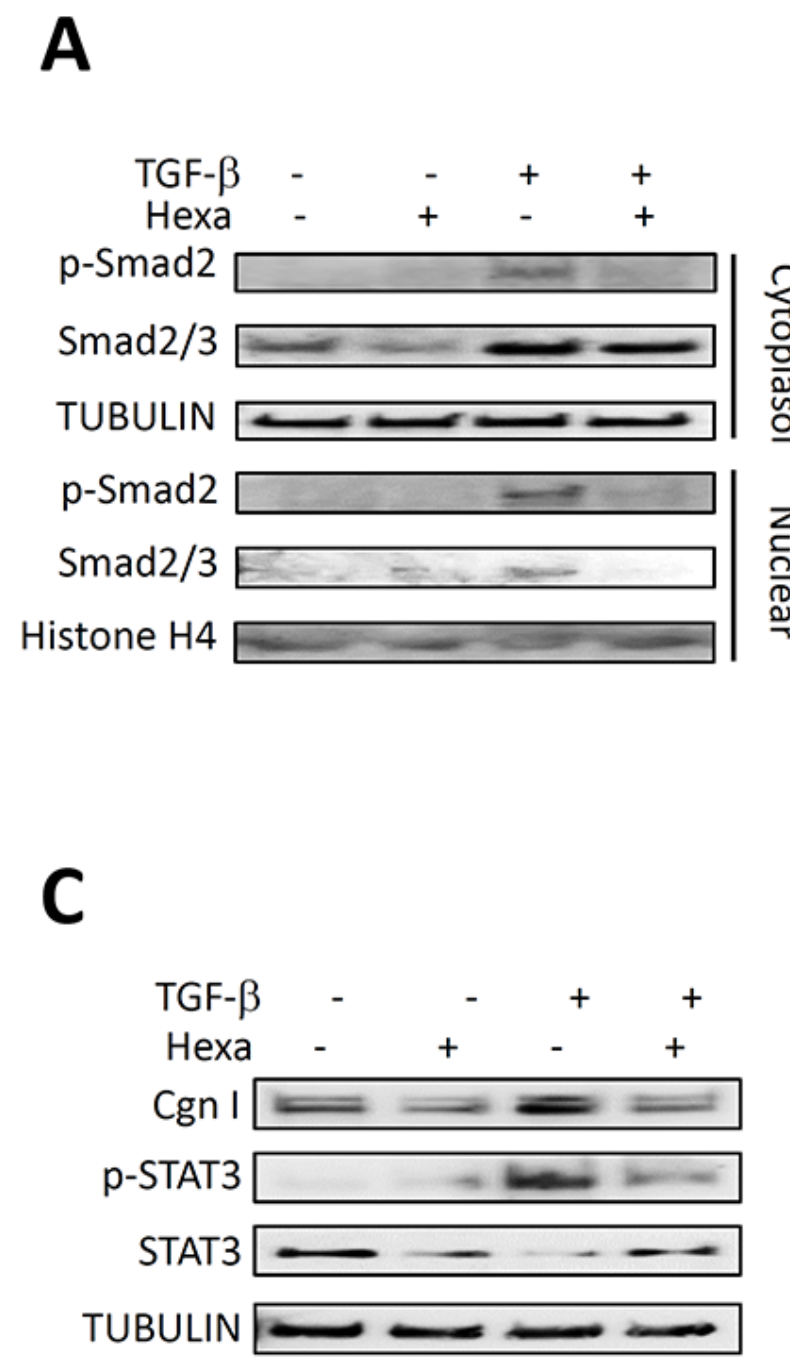

B

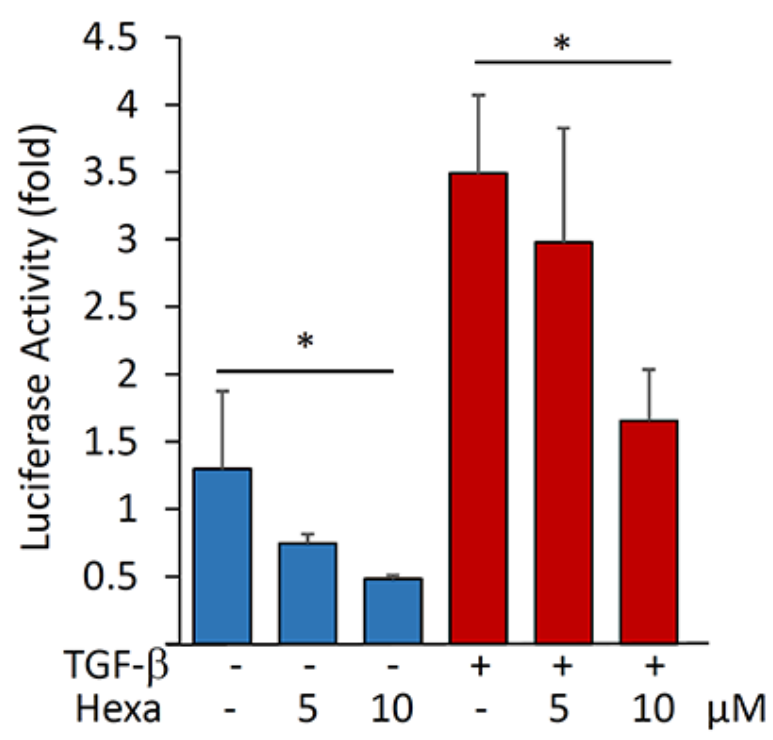

D

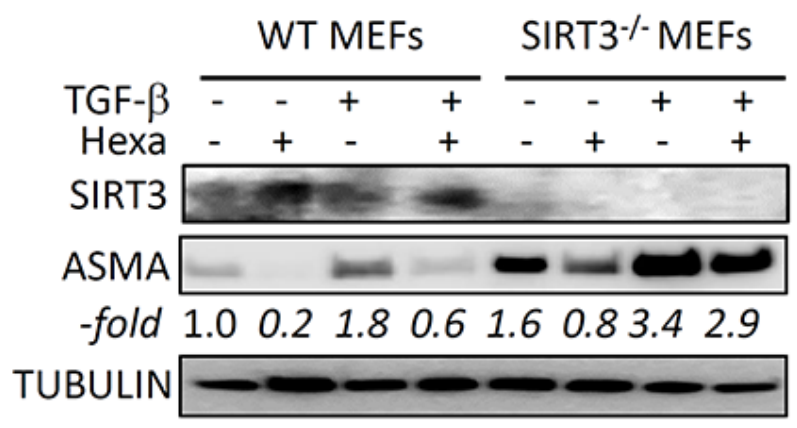

Figure 6: Hexafluoro abrogates fibrotic signaling. A., C. Lung fibroblasts were treated with $10 \mu \mathrm{M}$ hexafluoro in the presence or absence of TGF- $\beta(10 \mathrm{ng} / \mathrm{ml})$ for $120 \mathrm{~min}$. A. Cytoplasmic lysates and nuclear extracts were examined by Western analysis. Representative immunoblots. B. NHLF transiently transfected [SBE] 4 -luc along with SIRT3 or empty vector, were pre-incubated with $10 \mu \mathrm{M}$ hexafluoro for $30 \mathrm{~min}$, followed with TGF- $\beta(10 \mathrm{ng} / \mathrm{ml})$ for $24 \mathrm{~h}$. Whole cell lysates were analyzed for their luciferase activities. Results are the means \pm SD of triplicate experiments. ${ }^{*} p<0.05$. C. Whole cell lysates were examined by Western analysis. Representative immunoblots. Cgn I, Type I collagen. D. Confluent SIRT3-null (SIRT3 ${ }^{-/}$) and wild-type (WT) embryonic fibroblasts (MEFs) in parallel were preincubated with hexafluoro $(10 \mu \mathrm{M})$ for $30 \mathrm{~min}$, followed by TGF- $\beta(10 \mathrm{ng} / \mathrm{ml})$ for $24 \mathrm{~h}$. Whole cell lysates were examined by Western analysis. Band intensities normalized to tubulin are shown as -fold change below. 
Table 1: Clinical features of subjects donating skin biopsies used for SIRT3 immunohistochemistry.

\begin{tabular}{|l|l|l|l|l|l|}
\hline & $\begin{array}{l}\text { Age } \\
\text { yrs }\end{array}$ & gender & MRSS & Skin score at biopsy site & $\begin{array}{l}\text { Disease duration from 1st non-RP manifestation } \\
\text { (month) }\end{array}$ \\
\hline SSc 1 & 49 & F & 3 & 0 & 44 \\
\hline SSc 2 & 51 & M & 48 & 3 & 9 \\
\hline SSc 3 & 50 & F & 24 & 2 & 101 \\
\hline SSc 4 & 34 & F & 32 & 2 & 40 \\
\hline SSc 5 & 57 & F & 11 & 1 & 64 \\
\hline SSc 6 & 50 & F & 9 & 1 & 8 \\
\hline SSc 7 & 70 & F & 4 & 0 & 9 \\
\hline SSc 8 & 57 & F & 9 & 0 & 47 \\
\hline SSc 9 & 43 & F & 2 & 0 & 1 \\
\hline
\end{tabular}

MRSS, modified Rodnan skin score.

RP, Raynaud's phenomenon.

Skin score at biopsy site scored as follows: 0, normal thickness; 1, mild thickening; 2, moderate thickening; 3, severe thickening.

Table 2: Clinical features of subjects donating skin samples used for Ac-MnSOD immunofluorescence.

\begin{tabular}{|c|c|c|c|c|c|}
\hline & Age & gender & MRSS & Skin score at biopsy site & Disease duration from $1^{\text {st }}$ non-RP manifestation (month) \\
\hline SSc 10 & 67 & $\mathrm{~F}$ & 10 & 0 & 23 \\
\hline SSc 11 & 48 & M & 10 & 0 & 20 \\
\hline SSc 12 & 39 & $\mathrm{M}$ & 13 & 1 & 13 \\
\hline SSc 13 & 50 & $\mathrm{~F}$ & 14 & 1 & 23 \\
\hline SSc 14 & 52 & $\mathrm{~F}$ & 17 & 1 & 18 \\
\hline SSc 15 & 55 & $\mathrm{M}$ & 19 & 2 & 33 \\
\hline SSc 16 & 52 & $\mathrm{~F}$ & 24 & 2 & 37 \\
\hline SSc 17 & 58 & $\mathrm{~F}$ & 3 & 0 & 124 \\
\hline SSc 18 & 62 & $\mathrm{~F}$ & 3 & 0 & 140 \\
\hline SSc 19 & 30 & $F$ & 1 & 0 & 71 \\
\hline SSc 20 & 56 & $\mathrm{~F}$ & 6 & 0 & 30 \\
\hline $\mathrm{SSc} 21$ & 19 & $F$ & 0 & 0 & 16 \\
\hline $\mathrm{SSc} 22$ & 56 & $F$ & 3 & 0 & 104 \\
\hline $\mathrm{SSc} 23$ & 36 & $\mathrm{~F}$ & 2 & 0 & 151 \\
\hline $\mathrm{SSc} 24$ & 57 & F & 4 & 0 & 124 \\
\hline SSc 25 & 48 & $\mathrm{~F}$ & 5 & 0 & 240 \\
\hline SSc 26 & 56 & $\mathrm{M}$ & 2 & 0 & 28 \\
\hline SSc 27 & 31 & $\mathrm{~F}$ & 25 & 3 & 49 \\
\hline $\mathrm{SSc} 28$ & 51 & $\mathrm{~F}$ & 35 & 2 & 11 \\
\hline SSc 29 & 69 & $\mathrm{M}$ & 41 & 3 & 10 \\
\hline
\end{tabular}

MRSS, modified Rodnan skin score.

RP, Raynaud phenomenon.

Skin score at biopsy site scored as follows: 0 , normal thickness; 1 , mild thickening; 2 , moderate thickening; 3 , severe thickening.

progression. To address this possibility, we investigated SIRT3 expression and activation in SSc biopsies. Demographic and clinical features of the subjects are shown in Table 1 and Table 2. By immunohistochemistry, SIRT3 expression was detectable in normal skin biopsies, and appeared to be primarily localized in spindle-shaped fibroblastic cells, as well as in round cells throughout both the papillary and reticular dermis (Figure 8A). Additionally, prominent SIRT3 expression was evident in keratinocytes in the basal epidermis. While SSc skin biopsies generally demonstrated retention of SIRT3 in the basal epidermis, within the dermis substantially lower 
SIRT3 levels were observed in SSc compared to healthy control skin biopsies $(P<0.05)$. Of note, we found a significant negative correlation $\left(\mathrm{R}^{2}=0.5421 ; p=0.04\right)$ between the SIRT3 score (a semi-quantitative measure of SIRT3 levels) within the lesional skin and disease duration. In contrast, the modified Rodnan Skin Score (MRSS), a global measure of skin induration, showed no significant correlation with the skin SIRT3 score. To evaluate SIRT3 activity, levels of acetylated MnSOD, the prototypic SIRT3 substrate and a sensitive marker of SIRT activity, were examined. In sharp contrast to healthy skin biopsies, in SSc skin biopsies in a majority of interstitial cells within the dermis were immunopositve for Ac-MnSOD (Figure 8). Many of these cells were also positive for the myofibroblast marker $\alpha$ SMA, indicating attenuated SIRT3 activity within lesional myofibroblasts.
Lung biopsies from patients with SSc-ILD $(n=5$, Table 3 ) showed elevated expression of Ac-MnSOD within the fibrotic parenchyma compared to control lungs $(n=3)$. Alveolar macrophages showed detectable Ac-MnSOD expression in both SSc and control lungs.

When propagated in culture, explanted SSc fibroblasts maintain their activated phenotype even in the absence of exogenous stimuli, due in part to cellautonomous TGF- $\beta$ receptor phosphorylation and activity [34, 35]. To examine the cell-autonomous expression of SIRT3 in SSc, fibroblasts from dcSSc ( $n$ $=4)$ and matched healthy control $(n=4)$ skin biopsies were studied in parallel. By qPCR, significantly reduced SIRT3 expression was noted in SSc fibroblasts $(p=0.04$; Figure $8 \mathrm{D})$. Incubation of SSc fibroblasts $(n=4)$ with hexafluoro was able to attenuate $C O L 1 A 1, \alpha S M A$, and

A
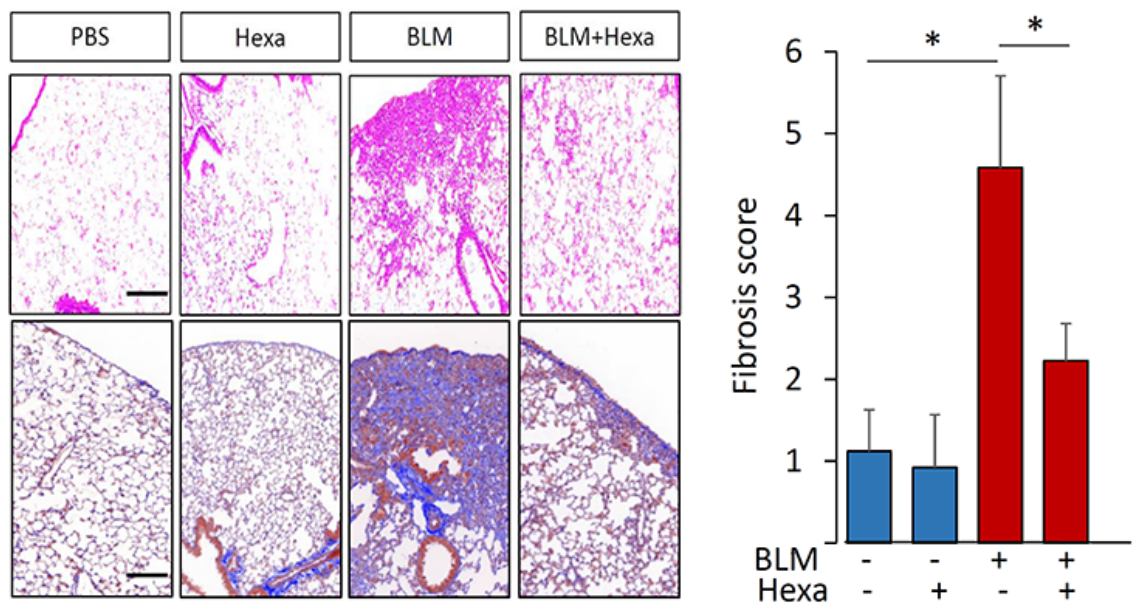

B
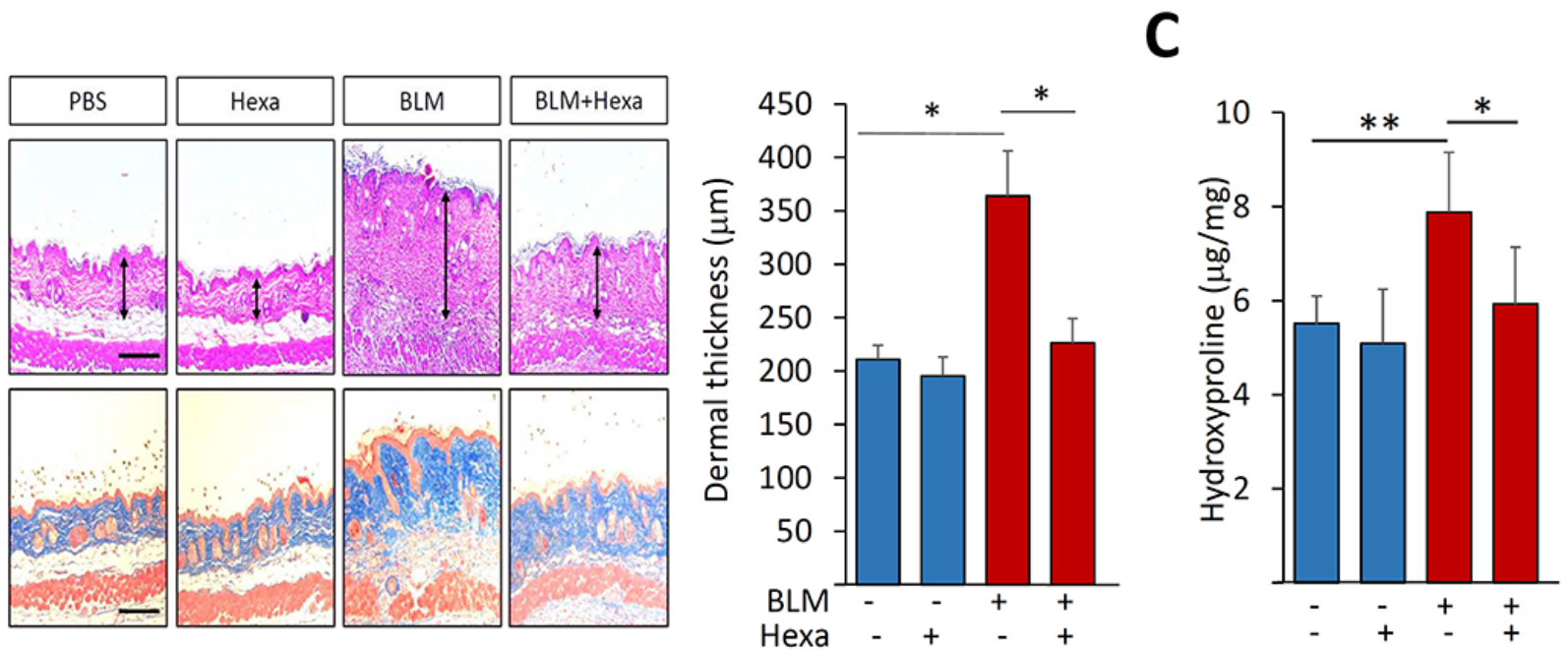

Figure 7: Hexafluoro treatment ameliorates bleomycin-induced organ fibrosis. Mice were treated with s.c. bleomycin (BLM) alone for 14 days, or in combination with i.p. hexafluoro, and sacrificed on day 28. A. Lung changes. Left panels, H\&E (upper) and Trichrome (lower) stain. Original magnification X 100. Right panels, lung fibrosis score determined as described in Materials and Methods. Bars are the means $\pm \mathrm{SD}$ of each group (4-7 mice/group). ${ }^{*} p<0.05$. B. Attenuation of dermal fibrosis. Left panels, H\&E (upper) and Trichrome (lower) stain. Bar $=50 \mu \mathrm{m}$. Right panel, quantification of dermal thickness. Bars represent means $\pm \mathrm{SEM}$ (4-7 mice per group). $* p<0.05$. C. Collagen content of the skin determined by hydroxyproline assays. Results are means $\pm \mathrm{SD}$ of duplicate determinations from 4-7 mice per group; ${ }^{*} p<0.05 ; * * p<0.01$ 
$F N^{E D A}$ expression (Table 4). We next examined Sirt3 expression in a murine model of scleroderma induced by s.c. bleomycin. Development of skin fibrosis in these mice was accompanied by significant down-regulation of Sirt3 within the lesional dermis (Figure 8E). Taken together, these results indicate that SIRT3 levels and activity are impaired in fibrotic lesions and explanted skin fibroblasts in SSc, as well as in a mouse model of scleroderma.

\section{DISCUSSION}

We show here that SIRT3, the major mitochondrial deacetylase, has potent cell-intrinsic anti-fibrotic effects, and its expression and activity are reduced in fibrotic tissues and explanted skin fibroblasts from patients with SSc. Hexafluoro, a novel synthetic honokiol derivative, induces SIRT3 expression and activity, and mitigates TGF$\beta$-induced mitochondrial oxidative stress and fibroblast stimulation, at least in part via stimulation of cellular SIRT3. Moreover, hexafluoro treatment has striking effects on mitigating skin and lung fibrosis in bleomycin-treated mice.

There is growing recognition of the importance of mitochondrial ROS as mediators of TGF- $\beta$ signal
A
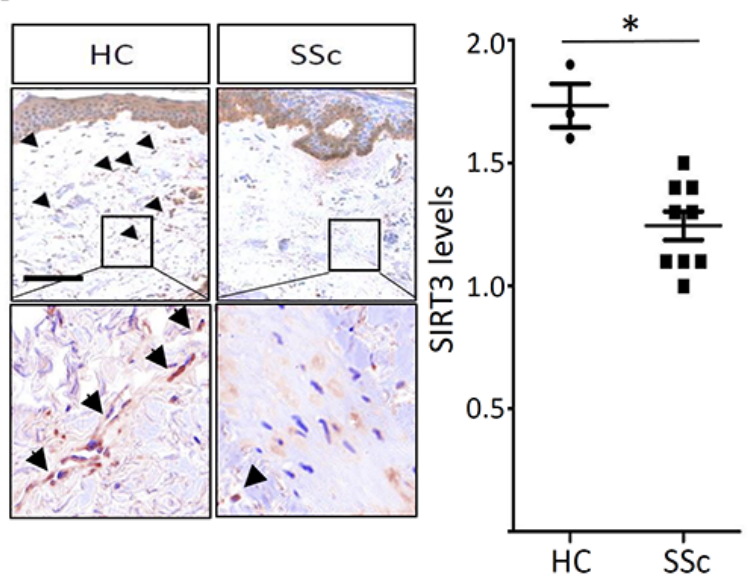

B

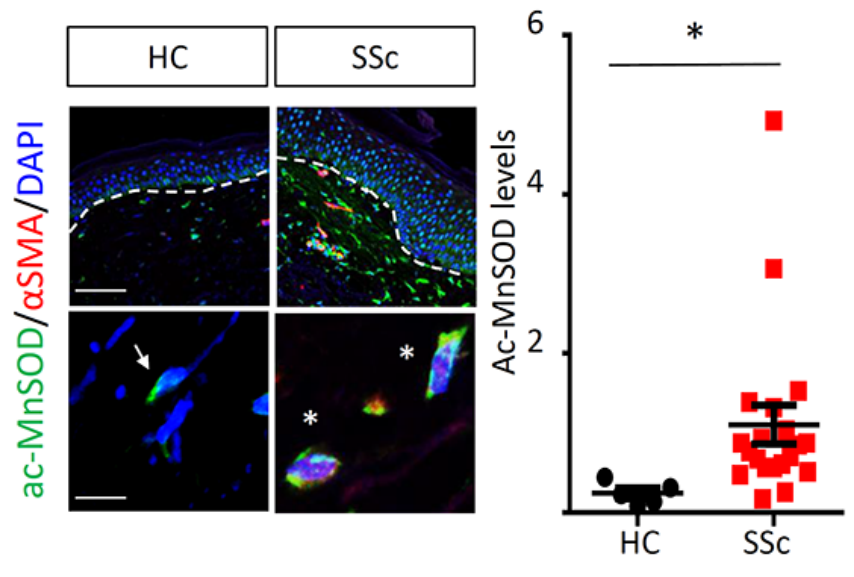

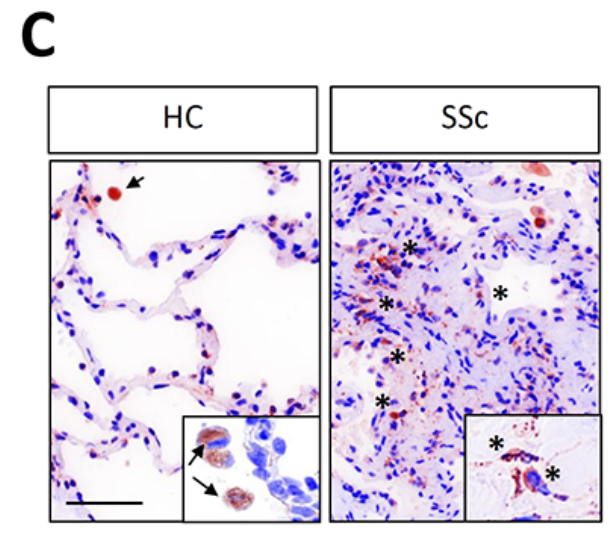

D

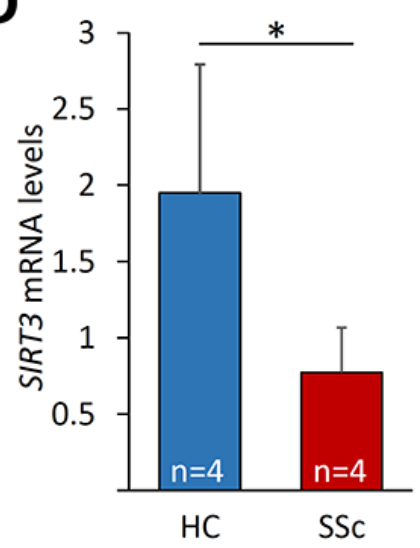

E

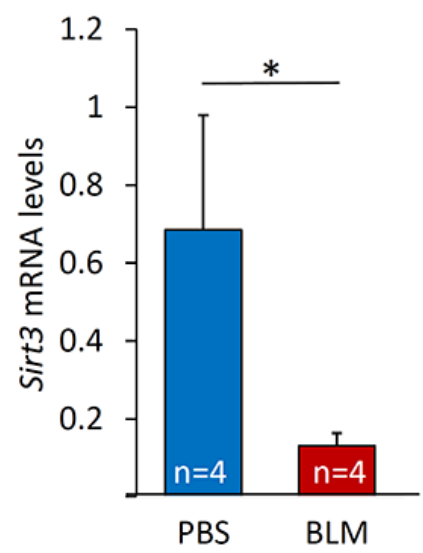

Figure 8: Attenuated SIRT3 expression and activity in SSc and in mouse models of fibrosis. A. Immmunohistochemistry of skin biopsies. Healthy control $(n=3)$ and dcSSc $(n=9)$ skin biopsies were evaluated using antibodies to SIRT3. Left panels, representative images. Arrowheads indicate immunopositive spindle-shaped cells within the dermis. Lower panels, higher magnification of boxed areas. Bar $=12.5 \mu \mathrm{m}$. Right panel, SIRT3 scores calculated as described in Materials and Methods. Bars represent the means \pm SD; $p<0.05$. B. Immunfluorescence. Left panels, immunostain of healthy control $(n=5)$ and SSc $(n=20)$ skin biopsies. Representative images. Green, Ac-MnSOD; red, $\alpha$ SMA; blue, DAPI. White dashed lines indicated epidermis-dermis junction. Arrows, Ac-MnSOD positive cells; star, Ac-MnSOD and $\alpha$ SMA double-positive cells. Bar $=100 \mu \mathrm{m}$ (upper panels) and $10 \mu \mathrm{m}$ (lower panels). Right panel, Ac-MnSOD levels. Each dot represents a biopsy. C. Immunohistochemistry of control $(n=3)$ and SSc $(n=6)$ lung biopsies using anti-Ac-MnSOD. Representative images. Inset, higher magnification. Arrow, Ac-MnSOD-positive alveolar macrophages; asterisk, Ac-MnSOD-positive stromal cells. Bar $=50 \mu \mathrm{m}$. D., E. Real-time qRT-PCR of lesional skin. Results, normalized with $G A P D H$, and the means \pm SD of each group. ${ }^{*} p<0.05$. D. RNA from confluent control $(n=4)$ and SSc $(n=4)$ skin fibroblasts was subjected to real-time qPCR. E. Mice treated with s.c. bleomycin (BLM; $n=4)$ or PBS $(n=4)$ for 14 days in parallel were sacrificed on day 28. RNA isolated from lesional skin was analyzed by qRT-PCR. Results, normalized with Gapdh, are means \pm SD of each group. ${ }^{*} p<0.05$. 
Table 3: Clinical features of subjects providing lung samples used for Ac-MnSOD immunofluorescence studies.

\begin{tabular}{|l|l|l|l|l|}
\hline & S-15-7641 & S15-47085 & S15-39544 & S16-11379 \\
\hline Age (yrs) & 61 & 42 & 58 & 53 \\
\hline Sex & F & M & F & M \\
\hline Specimen & Explant & Explant & Explant & Explant \\
\hline Duration of disease & Long standing & Long standing & Long standing & Long standing \\
\hline $\begin{array}{l}\text { Predominant } \\
\text { histological pattern }\end{array}$ & NSIP & NSIP and UIP & NSIP and UIP & UIP \\
\hline
\end{tabular}

NSIP, nonspecific interstitial pneumonitis; UIP, usual interstitial pneumonitis.

Table 4: Hexafluoro mitigates fibrotic gene expression in both normal SSc skin fibroblasts.

\begin{tabular}{|l|l|l|l|}
\hline Cell line & COL1A1* & $\boldsymbol{A S M A}^{*}$ & $\boldsymbol{F} \boldsymbol{N}^{\text {EDA }}$ * \\
\hline S1 & 0.30 & 0.36 & 0.47 \\
\hline S2 & 0.56 & 0.07 & 0.95 \\
\hline S3 & 0.35 & 0.37 & 0.27 \\
\hline S4 & 0.30 & 0.36 & 0.67 \\
\hline
\end{tabular}

*fold change in mRNA levels compared to untreated cultures.

Confluent cultures of low-passage SSc skin fibroblasts $(n=4)$ were incubated with hexafluoro $(10 \mu \mathrm{M})$ for $24 \mathrm{~h}$. Results of real-time qPCR, normalized with GAPDH.

transduction in the context of fibrogenesis [29,36]. SIRT3 plays critical role in regulating mtROS production [25, 37]. Here we examined the regulation of SIRT3 expression and function by TGF- $\beta$, the major fibrogenic growth factor implicated in fibrosis $[4,31]$. Our results showed that expression of SIRT3 is suppressed by TGF- $\beta$ treatment of explanted skin and lung fibroblasts. A series of cellbased assays with gain- and loss-of-function were carried out to delineate the mechanism of SIRT3 effects in the context of fibrogenesis. Ectopic SIRT3 attenuated TGF- $\beta$ induced stimulation of collagen synthesis and fibrotic responses in both lung and skin fibroblasts, while RNAi of cellular SIRT3 resulted in enhanced fibrotic responses. The anti-fibrotic effects of SIRT3 involved disruption of canonical TGF- $\beta$ signal transduction and Smad-dependent transcription. Furthermore, we found that both tissue expression and function of SIRT3 were substantially reduced in skin and lung biopsies from SSc patients, as well as in mice with bleomycin-induced skin fibrosis. Taken together, these findings suggest that SIRT3 might have an important negative regulatory function to check TGF- $\beta$-dependent fibrotic responses in fibroblasts, and its deficiency in SSc, possibly reflecting its down-regulation by TGF- $\beta$ within the fibrotic milieu, might play a causal role in the persistence of fibrosis in these tissues. We did not demonstrate correlation between SIRT3 expression levels in the skin and the extent of skin fibrosis, as measured by MRSS. Whether reduced SIRT3 expression/ activity might correlate with progression of skin fibrosis over time, suggesting a potential role as a predictive biomarker, remains to be determined in longitudinal analyses.

Sirtuins belong to an evolutionary conserved family of $\mathrm{NAD}^{+}$-dependent protein lysine deacylases that play key roles in the regulation of metabolism, stress responses, and aging processes. Honokiol, a naturallyoccuring biphenolic compound, has pleiotropic effects and potential pharmacological utility [38]. To further enhance honokiol's drug-like properties, recent efforts focused on modification resulting in the novel compound hexafluoro [24]. We now show that hexafluoro induced a rapid increase in SIRT3 mRNA and protein expression, and rescued SIRT3 expression even in the presence of TGF- $\beta$. Stimulation of SIRT3 was accompanied by attenuation of cytosolic and mitochondrial ROS, and restoration of perturbed mitochondrial membrane potential. These mitochondrial-protective effects of hexafluoro are likely to be related to induction of cellular SIRT3, which serves as the major mitochondrial deacetylase to reduce oxidative stress $[12,39]$. However, the effects of honokiol derivatives appear to be cell-type specific, and ROS production was enhanced by honokiol derivatives, including hexafluoro, in melanoma cell lines [24]. In our studies, treatment of both lung and skin fibroblasts with hexafluoro attenuated TGF- $\beta$ induced pro-fibrotic gene expression and myofibroblast differentiation, contractility and migration, and mitigated constitutive fibrotic gene expression in SSc fibroblast. These effects were associated with attenuated Smad signaling, indicating that hexafluoro induced factors that antagonize receptor-dependent Smad activity. Activation of Stat3, an important mediator of cytokine signaling linked to fibrosis, was also attenuated [40]. Whether impaired Stat3 activation in fibroblasts treated with hexafluoro is a direct effect, or is mediated via endogenous SIRT3, remains to be addressed. Treatment of mice with hexafluoro mitigated both lung and skin fibrosis induced by bleomycin. The role of attenuated mitochondrial oxidative stress and altered autoimmunity, 
in these anti-fibrotic effects and if they remain to be established.

In summary, we show here that the mitochondrial deacetylase SIRT3 has cell-intrinsic potent anti-fibrotic properties in fibroblasts, and its expression and activity are impaired in SSc skin and lung biopsies. A recent study demonstrated comparable reduced SIRT3 expression in the explanted lungs from patients with SSc-ILD, providing further evidence for the potential role of impaired SIRT3 function in the disease [41]. Furthermore, the novel synthetic honokiol derivative hexafluoro has SIRT3 agonist activities coupled with inhibitory effects on mitochondrial ROS generation and TGF- $\beta$-induced fibrotic responses in fibroblasts, and on experimental organ fibrosis in mice. The results implicate, for the first time, SIRT3 deficit as a pathogenic mechanism underlying fibrosis in SSc, and suggest that hexafluoro might have therapeutic potential via restoring SIRT3 in the treatment for fibrotic conditions.

\section{MATERIALS AND METHODS}

\section{Human subjects}

The protocols for human tissue procurement (skin and lung) were approved by the Institutional Review Boards for Human Studies at Northwestern University. All patients fulfilled the American College of Rheumatology/ European league Against Rheumatism 2013 classification criteria for SSc [42]. Skin biopsies were performed after obtaining written informed consent. Lung tissues were obtained from five SSc patients undergoing lung transplantation for advanced ILD. The demographics and clinical characteristics of patients were listed in Tables 1,2 and 3 .

\section{Bleomycin-induced scleroderma in the mouse}

Animal protocols were institutionally approved by the Animal Care and Use Committees of Northwestern University. Eight to ten week-old female C57/BL6J mice (The Jackson Laboratory, Bar Harbor, ME) were randomized to receive vehicle, bleomycin (APP, Schaumburg, IL), or a combination of hexafluoro and bleomycin (4-7 mice/group). Hexafluoro was synthesized and purified by column chromatography as described [24]. Mice were given daily subcutaneous (s.c.) injections of PBS or $10 \mathrm{mg} / \mathrm{kg}$ bleomycin for 14 days [43]. Hexafluoro was given by daily intraperitoneal (i.p.) injections five times/week at a dose of $70 \mathrm{mg} / \mathrm{kg}$ in a cocktail made by dissolving $16 \mathrm{mg}$ of compound into $100 \mu \mathrm{l}$ of absolute ethanol. The ethanol-compound solutions were then added to $20 \%$ soy-fat Intralipid (Frensenius Kabi, Lake Zurich, IL) and vortexed vigorously[24]. Mice were sacrificed on day 28, and lesional skin and lung were harvested. Each experimental group included 4-7 mice. Full-thickness skin sections were taken from injected back region, and lung sections were taken from the right lungs. Tissues were fixed in 10\% formaldehyde for 48 or $96 \mathrm{~h}$ respectively, and embedded in paraffin. Sections were stained with hematoxylin and eosin (H\&E) or Masson's trichrome. Dermal thickness, defined as the distance from the epidermal-dermal junction to the junction between the dermis and subcutaneous fat, was determined at five random locations per section [28]. All sections were examined independently by two investigators in a blinded manner. Severity of lung fibrosis was graded on a scale of 0 to 8 by examining five randomly chosen fields of the same right lobe at $100 \times$ magnification as follows: grade 0, normal lung; grade 1, minimal fibrous thickening of alveolar or bronchiolar walls; grade 3, moderate thickening of walls without obvious damage to lung architecture; grade 5, increased fibrosis with definite damage to lung structure and formation of fibrous bands or small fibrous masses; grade 7, severe distortion of structure and large fibrous areas; and grade 8, total fibrous obliteration. Sections were scored independently by two investigators in a blinded manner. Collagen accumulation was determined by measuring hydroxyproline content in half of 8-mm skin biopsy samples [44]. Results are expressed as total hydroxyproline per mg tissue.

\section{Fibroblast cultures}

Primary cultures of dermal fibroblasts were established by explanation from foreskins from healthy newborns or from skin biopsies from SSc patients or age-matched healthy controls [43]. Normal adult lung fibroblast were purchased from LONZA (Walkersville, $\mathrm{MD})$. Cells were incubated at $37^{\circ} \mathrm{C}$ in an atmosphere of $5 \% \mathrm{CO}_{2}$ in Dulbecco's modified Eagle's medium (DMEM) supplemented with $10 \%$ fetal bovine serum, $1 \%$ vitamins, $1 \%$ penicillin/streptomycin, and $2 \mathrm{mM} \mathrm{L-glutamine} \mathrm{(all}$ from BioWhittaker) for skin fibroblasts and FGM-2 bullet kit (LONZA) for NHLF, respectively. Cells were used between passage 4 and 8 . At early confluence, cultures were incubated with hexafluoro in the presence or absence of TGF- $\beta 2$ (10 ng/ml) (PeproTech, Rocky Hill, NJ). Cell toxicity was evaluated using lactate dehydrogenase cytotoxicity assay kits (BioVision, Milpitas, CA), and cell viability by trypan blue dye exclusion.

\section{Gel contraction and cell migration assays}

To assess the effects of hexafluoro on cell contraction, lung fibroblasts were seeded in type I collagen gels (BD Bioscience, San Jose, CA) that were then incubated in medium with or without TGF- $\beta 2$ (10 $\mathrm{ng} / \mathrm{ml}$ ) and hexafluoro for up to $72 \mathrm{~h} \mathrm{[43].} \mathrm{After} 72 \mathrm{~h}$, gel 
diameters were determined. Modulation of cell migration by hexafluoro was evaluated by in vitro wound healing assays. Briefly, confluent monolayers of fibroblasts were incubated in serum-free medium with hexafluoro for $12 \mathrm{~h}$ in the presence of $10 \mu \mathrm{g} / \mathrm{ml}$ Mitomycin C (Sigma, St Louis, MO), and scratch wounds were created using standard p1000 pipette tips [43]. Cell migration was then monitored by phase-contrast microscopy for up to $48 \mathrm{~h}$. Gap width was determined at 3 different sites per sample at indicated intervals.

\section{Plasmids, small interfering RNA (siRNA), and transient transfection assays}

Small interfering RNAs specific for SIRT3 and scrambled control siRNA were purchased from Santa Cruz Biotechnology (Dallas, TX). The plasmid $\mathrm{SBE}_{4}-\mathrm{TK}-$ Luc contains four copies of the consensus Smad-binding element linked to thymidine kinase and luciferase genes [43]. Subconfluent cultures were transfected with SIRT3 expression vector (OriGene, Rockville, MD) or empty vector, $\mathrm{SBE}_{4}-\mathrm{TK}$-Luc or siRNA (Santa Cruz) for $48 \mathrm{~h}$, followed by incubation with or without TGF- $\beta 2$ (10 ng/ $\mathrm{ml}$ ) and hexafluoro for $24 \mathrm{~h}$. Cultures were harvested and whole-cell lysates were assayed for their luciferase activities using the Dual-Luciferase Reporter Assay system (Promega, Madison, WI) [43]. The reporter vector pRL-TK Renilla luciferase (pRL-TK Luc) was used in each experiment as an internal control, and experiments were repeated at least 3 times.

\section{Evaluation of ROS generation and mitochondrial membrane potential changes}

At early confluence fibroblasts seeded on cover slips were incubated in media with or without TGF- $\beta 2$ (10 ng/ $\mathrm{ml})$ or hexafluoro $(10 \mu \mathrm{M})$ for $24 \mathrm{~h}$. Mitochondrial ROS levels were determined using MitoSOX Red superoxide indicator reagent that concentrates within mitochondria and fluoresces red when oxidized by ROS. Cytosolic ROS production was assessed using CellROX green reagent (both from Invirogen). Fluorescence was determined under a confocal microscope (Nikon C2+, Nikon Tokyo, Japan) at $495 \mathrm{~nm}$ (green), $565 \mathrm{~nm}$ (red) and $400 \mathrm{~nm}$ (blue), and intensity was quantitated using Image J. In order to assess changes in mitochondrial membrane potential, tetramethylrhodamine (TMRM) mitochondrial membrane potential-dependent cationic red dye (Thermo Scientific) was used [30].

\section{RNA isolation and quantitative real-time PCR}

Total RNA was isolated from explanted fibroblasts using Quick RNA Miniprep (Zymo Research), and quantitative PCR was performed [43]. Levels of mRNA normalized to $G A P D H$ levels in each sample were determined by calculating $2^{-\Delta \Delta} C_{t}$. Dissociation analysis for each primer pair and reaction was performed to verify specific amplification.

\section{Western analysis}

At the end of the experiments, cells were lysed with passive protein lysis buffer (Promega, Fitchburg, WI), and $5-15 \mu \mathrm{g}$ total proteins were applied to $4-15 \%$ SDSpolyacrylamide gel. After electrophoresis, the proteins were transferred to PVDF membranes, followed by blocking in the buffer containing $10 \%$ fat-free dry milk. The membranes were probed with indicated first antibodies overnight, and then washed three times in TBS-T and incubated with HRP-conjugated secondary antibodies for $60 \mathrm{~min}$ and visualized using chemiluminescence ECL reagent (Sigma). The following antibodies were used: anti-tubulin, anti-SIRT3, anti-SIRT1, anti-histone H4, anti$\mathrm{Fn}^{\mathrm{EDA}}$, anti- $\alpha \mathrm{SMA}$ (Sigma), anti- type I collagen (Southern Bio), anti-phospho-Smad2, anti-Smad2/3, anti-STAT3, and anti-phospho-STAT3 (Cell Signaling). Membranes were developed using Fuji scanner and analyzed by AIDA software (Fuji, Inc., Tokyo, Japan).

\section{Immunohistochemistry and immunofluorescence}

Skin and lung biopsies from healthy adults, nonSSc subjects and SSc patients (Tables 1 and 2) were examined by immunohistochemistry. Briefly, 4- $\mu \mathrm{m}$ paraffin-embedded sections were incubated overnight with primary antibodies to SIRT3 (Cell Signaling Technology, San Antonio, TX) or Ac-MnSOD (Abcam, Cambridge, MA) overnight, followed by incubation with biotinylated donkey anti-rabbit secondary antibodies and streptavidin-linked alkaline phosphatase (AP) (Jackson ImmunoResearch, West Grove, PA) or AlexaFlouro conjugated secondary antibodies (Invitrogen). Levels of SIRT3 were evaluated using a semi-quantitative approach. Immunostaining intensities were determined by two independent blinded observers by scoring $\geq 60$ individual fibroblastic cells in 3 randomly chosen highpower fields per biopsy throughout the dermis as follows: $0=$ no detectable staining: 1 = faint staining: 2 = moderate staining: 3 = strong staining. Levels of Ac-MnSOD in dermal cells were quantitated by measuring fluorescence intensity using ImageJ, and the results were normalized with DAPI fluorescence intensity. SIRT3 and ac-MnSOD scores were calculated by taking the means of fluorescence intensities. Isotype IgG was used as negative controls (Suppl. Fig 3). To evaluate cellular levels of type I collagen and $\alpha$ SMA, confluent fibroblasts were pre-incubated with $10 \mu \mathrm{M}$ hexafluoro for $60 \mathrm{~min}$ prior to incubation with $10 \mathrm{ng} / \mathrm{mL}$ TGF- $\beta 2$. Twenty-four hours later, cells were 
fixed, incubated with primary antibodies against type I collagen (1:200; Southern Biotech, Birmingham, AL) or $\alpha$-SMA (1:500; Sigma Aldrich) overnight, followed by incubation with Alexa Fluor 594-conjugated donkey antigoat (Invitrogen) or Alexa Fluor 488-conjugated chicken anti-mouse antibodies for $120 \mathrm{~min}$. Nuclei were identified by DAPI staining (1:5000). Nonimmune IgG was used as a negative control in each experiment. Following stringent washing, slides were examined under Nikon C2+ confocal microscope. Each experiment was repeated at least 3 times with consistent results.

\section{Statistical analysis}

All values are expressed as the mean $\pm \mathrm{SD}$. At least three replicates were applied for each experiment. Statistical differences among groups were determined using either Student's $t$ test or one-way ANOVA. In the animal and human studies, differences between the groups were evaluated using non-parametric Mann-Whitney U test. $P$ values of less than 0.05 were considered statistically significant.

\section{ACKNOWLEDGMENTS}

We thank Drs. Warren G. Tourtellotte and Renea Jablonski (Northwestern University Feinberg School of Medicine) for helpful discussions, and members of the Northwestern Scleroderma Program, Dr Monique E Hinchcliff and the staffs of the Cell Imaging Facility and Mouse Histology and Phenotyping Laboratory for invaluable technical assistance. Supported by grants from the National Institutes of Health (AR-49025 to JV, HL-111455 and HL-117041 to MPG, NIH-ES020357 to DWK), National Scleroderma Foundation (JV) and VA Merit (DWK).

\section{CONFLICTS OF INTEREST}

There is no conflict of interest.

\section{Author contributions}

JV and JLA conceived the study. KA and JW designed and performed the research, analyzed and interpreted data, and wrote and edited the manuscript. MB carried out some of the experiments. MYB and PC assisted with selected experiments. KA, JW, MPG, JLA, PC, DWK and JV interpreted data, and prepared the manuscript.

\section{REFERENCES}

1. Wynn TA and Ramalingam TR. Mechanisms of fibrosis: therapeutic translation for fibrotic disease. Nature medicine.
2012; 18(7):1028-1040.

2. Gabrielli A, Avvedimento EV and Krieg T. Scleroderma. The New England journal of medicine. 2009; 360(19):19892003.

3. Topal AA and Dhurat RS. Scleroderma therapy: clinical overview of current trends and future perspective. Rheumatol Int. 2013; 33(1):1-18.

4. Bhattacharyya S, Wei J and Varga J. Understanding fibrosis in systemic sclerosis: shifting paradigms, emerging opportunities. Nat Rev Rheumatol. 2012; 8(1):42-54.

5. Selman $\mathrm{M}$ and Pardo A. Revealing the pathogenic and aging-related mechanisms of the enigmatic idiopathic pulmonary fibrosis. an integral model. American journal of respiratory and critical care medicine. 2014; 189(10):11611172.

6. Hu T, Ramachandrarao SP, Siva S, Valancius C, Zhu Y, Mahadev K, Toh I, Goldstein BJ, Woolkalis M and Sharma $\mathrm{K}$. Reactive oxygen species production via NADPH oxidase mediates TGF-beta-induced cytoskeletal alterations in endothelial cells. Am J Physiol Renal Physiol. 2005; 289(4):F816-825.

7. North BJ and Verdin E. Sirtuins: Sir2-related NADdependent protein deacetylases. Genome Biol. 2004; 5(5):224.

8. Michan S and Sinclair D. Sirtuins in mammals: insights into their biological function. Biochem J. 2007; 404(1):1-13.

9. Saunders LR and Verdin E. Sirtuins: critical regulators at the crossroads between cancer and aging. Oncogene. 2007; 26(37):5489-5504.

10. Lavu S, Boss O, Elliott PJ and Lambert PD. Sirtuins--novel therapeutic targets to treat age-associated diseases. Nat Rev Drug Discov. 2008; 7(10):841-853.

11. Brown K, Xie S, Qiu X, Mohrin M, Shin J, Liu Y, Zhang D, Scadden DT and Chen D. SIRT3 reverses aging-associated degeneration. Cell Rep. 2013; 3(2):319-327.

12. Tao R, Vassilopoulos A, Parisiadou L, Yan Y and Gius D. Regulation of MnSOD enzymatic activity by Sirt3 connects the mitochondrial acetylome signaling networks to aging and carcinogenesis. Antioxidants \& redox signaling. 2014; 20(10):1646-1654.

13. Sargent JL, Milano A, Bhattacharyya S, Varga J, Connolly MK, Chang HY and Whitfield ML. A TGFbeta-responsive gene signature is associated with a subset of diffuse scleroderma with increased disease severity. The Journal of investigative dermatology. 2010; 130(3):694-705.

14. Pillai VB, Samant S, Sundaresan NR, Raghuraman H, Kim G, Bonner MY, Arbiser JL, Walker DI, Jones DP, Gius D and Gupta MP. Honokiol blocks and reverses cardiac hypertrophy in mice by activating mitochondrial Sirt3. Nature communications. 2015; 6:6656.

15. Singh KK. Mitochondria damage checkpoint, aging, and cancer. Annals of the New York Academy of Sciences. 2006; 1067:182-190.

16. Bresciani G, da Cruz IB and Gonzalez-Gallego J. 
Manganese superoxide dismutase and oxidative stress modulation. Advances in clinical chemistry. 2015; 68:87130.

17. Chen Y, Zhang J, Lin Y, Lei Q, Guan KL, Zhao S and Xiong Y. Tumour suppressor SIRT3 deacetylates and activates manganese superoxide dismutase to scavenge ROS. EMBO reports. 2011; 12(6):534-541.

18. Qiu X, Brown K, Hirschey MD, Verdin E and Chen D. Calorie restriction reduces oxidative stress by SIRT3mediated SOD2 activation. Cell metabolism. 2010; 12(6):662-667.

19. Tao R, Coleman MC, Pennington JD, Ozden O, Park SH, Jiang H, Kim HS, Flynn CR, Hill S, Hayes McDonald W, Olivier AK, Spitz DR and Gius D. Sirt3-mediated deacetylation of evolutionarily conserved lysine 122 regulates MnSOD activity in response to stress. Molecular cell. 2010; 40(6):893-904.

20. Chao LK, Liao PC, Ho CL, Wang EI, Chuang CC, Chiu HW, Hung LB and Hua KF. Anti-inflammatory bioactivities of honokiol through inhibition of protein kinase $\mathrm{C}$, mitogenactivated protein kinase, and the NF-kappaB pathway to reduce LPS-induced TNFalpha and NO expression. J Agric Food Chem. 2010; 58(6):3472-3478.

21. Bai X, Cerimele F, Ushio-Fukai M, Waqas M, Campbell PM, Govindarajan B, Der CJ, Battle T, Frank DA, Ye K, Murad E, Dubiel W, Soff G and Arbiser JL. Honokiol, a small molecular weight natural product, inhibits angiogenesis in vitro and tumor growth in vivo. The Journal of biological chemistry. 2003; 278(37):35501-35507.

22. Shen JL, Man KM, Huang PH, Chen WC, Chen DC, Cheng YW, Liu PL, Chou MC and Chen YH. Honokiol and magnolol as multifunctional antioxidative molecules for dermatologic disorders. Molecules. 2010; 15(9):6452-6465.

23. Woodbury A, Yu SP, Wei L and Garcia P. Neuromodulating effects of honokiol: a review. Front Neurol. 2013; 4:130.

24. Bonner MY, Karlsson I, Rodolfo M, Arnold RS, Vergani $\mathrm{E}$ and Arbiser JL. Honokiol bis-dichloroacetate (Honokiol DCA) demonstrates activity in vemurafenib-resistant melanoma in vivo. Oncotarget. 2016; 7:12857-68. doi: 10.18632/oncotarget.7289.

25. Kim SJ, Cheresh P, Jablonski RP, Williams DB and Kamp DW. The Role of Mitochondrial DNA in Mediating Alveolar Epithelial Cell Apoptosis and Pulmonary Fibrosis. International journal of molecular sciences. 2015; 16(9):21486-21519.

26. Sundaresan NR, Gupta M, Kim G, Rajamohan SB, Isbatan A and Gupta MP. Sirt3 blocks the cardiac hypertrophic response by augmenting Foxo3a-dependent antioxidant defense mechanisms in mice. The Journal of clinical investigation. 2009; 119(9):2758-2771.

27. Macconi D, Perico L and Benigni A. Mitochondrial Dynamics Is Linked to Longevity and Protects from EndOrgan Injury: The Emerging Role of Sirtuin 3. Antioxidants
\& redox signaling. 2016.

28. Wu M, Melichian DS, Chang E, Warner-Blankenship M, Ghosh AK and Varga J. Rosiglitazone abrogates bleomycininduced scleroderma and blocks profibrotic responses through peroxisome proliferator-activated receptor-gamma. The American journal of pathology. 2009; 174(2):519-533.

29. Jain M, Rivera S, Monclus EA, Synenki L, Zirk A, Eisenbart J, Feghali-Bostwick C, Mutlu GM, Budinger GR and Chandel NS. Mitochondrial reactive oxygen species regulate transforming growth factor-beta signaling. The Journal of biological chemistry. 2013; 288(2):770-777.

30. Negmadjanov U, Godic Z, Rizvi F, Emelyanova L, Ross G, Richards J, Holmuhamedov EL and Jahangir A. TGF-beta1mediated differentiation of fibroblasts is associated with increased mitochondrial content and cellular respiration. PloS one. 2015; 10(4):e0123046.

31. Varga $\mathbf{J}$ and Pasche B. Transforming growth factor beta as a therapeutic target in systemic sclerosis. Nature reviews Rheumatology. 2009; 5(4):200-206.

32. Lin L, Amin R, Gallicano GI, Glasgow E, Jogunoori W, Jessup JM, Zasloff M, Marshall JL, Shetty K, Johnson L, Mishra L and He AR. The STAT3 inhibitor NSC 74859 is effective in hepatocellular cancers with disrupted TGF-beta signaling. Oncogene. 2009; 28(7):961-972.

33. Wu M and Varga J. In perspective: murine models of scleroderma. Current rheumatology reports. 2008; 10(3):173-182.

34. Mori Y, Chen SJ and Varga J. Expression and regulation of intracellular SMAD signaling in scleroderma skin fibroblasts. Arthritis and rheumatism. 2003; 48(7):19641978.

35. Jinnin M, Ihn H, Mimura Y, Asano $\mathrm{Y}$ and Tamaki K. Involvement of the constitutive complex formation of c-Ski/SnoN with Smads in the impaired negative feedback regulation of transforming growth factor beta signaling in scleroderma fibroblasts. Arthritis and rheumatism. 2007; 56(5):1694-1705.

36. Kudryavtseva AV, Krasnov GS, Dmitriev AA, Alekseev BY, Kardymon OL, Sadritdinova AF, Fedorova MS, Pokrovsky AV, Melnikova NV, Kaprin AD, Moskalev AA and Snezhkina AV. Mitochondrial dysfunction and oxidative stress in aging and cancer. Oncotarget. 2016; 7:44879-44905. doi: 10.18632/oncotarget.9821.

37. Pillai VB, Bindu S, Sharp W, Fang YH, Kim G, Gupta M, Samant S and Gupta MP. Sirt3 protects mitochondrial DNA damage and blocks the development of doxorubicin-induced cardiomyopathy in mice. American journal of physiology Heart and circulatory physiology. 2016; 310(8):H962-972.

38. Arora S, Singh S, Piazza GA, Contreras CM, Panyam J and Singh AP. Honokiol: a novel natural agent for cancer prevention and therapy. Current molecular medicine. 2012; 12(10):1244-1252.

39. Yu W, Dittenhafer-Reed KE and Denu JM. SIRT3 protein deacetylates isocitrate dehydrogenase 2 (IDH2) 
and regulates mitochondrial redox status. The Journal of biological chemistry. 2012; 287(17):14078-14086.

40. Pedroza M, Le TT, Lewis K, Karmouty-Quintana H, To S, George AT, Blackburn MR, Tweardy DJ and Agarwal SK. STAT-3 contributes to pulmonary fibrosis through epithelial injury and fibroblast-myofibroblast differentiation. FASEB journal. 2016; 30(1):129-140.

41. Sosulski ML, Gongora R, Feghali-Bostwick C, Lasky JA and Sanchez CG. Sirtuin 3 Deregulation Promotes Pulmonary Fibrosis. J Gerontol A Biol Sci Med Sci. 2016.

42. van den Hoogen F, Khanna D, Fransen J, Johnson SR, Baron M, Tyndall A, Matucci-Cerinic M, Naden RP, Medsger TA, Jr., Carreira PE, Riemekasten G, Clements PJ, Denton CP, et al. 2013 classification criteria for systemic sclerosis: an American College of Rheumatology/European League against Rheumatism collaborative initiative. Arthritis and rheumatism. 2013; 65(11):2737-2747.
43. Wei J, Zhu H, Komura K, Lord G, Tomcik M, Wang W, Doniparthi S, Tamaki Z, Hinchcliff M, Distler JH and Varga J. A synthetic PPAR-gamma agonist triterpenoid ameliorates experimental fibrosis: PPAR-gammaindependent suppression of fibrotic responses. Ann Rheum Dis. 2014; 73(2):446-454.

44. Wei J, Ghosh AK, Chu H, Fang F, Hinchcliff ME, Wang J, Marangoni RG and Varga J. The Histone Deacetylase Sirtuin 1 Is Reduced in Systemic Sclerosis and Abrogates Fibrotic Responses by Targeting Transforming Growth Factor beta Signaling. Arthritis Rheumatol. 2015; 67(5):1323-1334. 